\title{
AN IMAGE-BASED MULTIMEDIA DATABASE AND EFFICIENT DETECTION THOUGH FEATURES
}

\author{
KHURRAM EJAZ*1, Dr. MOHD SHAFry MOHD RAHIM ${ }^{1}$, Dr. AMJAD REHMAN ${ }^{2}$, ENGR FARHAN EJAZ ${ }^{3}$ \\ ${ }^{1}$ Faculty of Computing, University of Technology, Malaysia \\ ${ }^{2}$ Faculty of CCIS/ Al-Yamamah University, Riyadh, Kingdom of Saudi Arabia. \\ ${ }^{3}$ Faculty of Engineering and Technology, Institute of Engineering and Technology, Pakistan \\ Email: kejaz2@live.utm.my
}

\begin{abstract}
Accurate feature detection during Image retrieval is important, data retrieves through image retrieval methods like CBIR and CBIR higher dimension data also need storage and access through different methods, content-based Image retrieval uses query like query by feature and query by example. More focus has made on accurate feature detection because need accurate feature retrieval. In simple words objectives are, to develop methods with sequence to classify features with normalization for efficient image retrieval from bulk dataset and also to improve method for local and global feature retrieval with automatic feature detection along accuracy. After study of different detection-based system, a methodology has been proposed which improves retrieval based on feature detection and feature detection had been improve with combination $D W T+P C A+K S V M$ (polygon kernel $+R B F$ kernel + Linear Kernel).
\end{abstract}

Keywords: Image content; Content bases Image retrieval; Semantics; Query by feature, Query by example.

1. Introduction. Image detection is very essential part for retrieval of image, retrieval of image is possible base on features. Image retrieval is performed from multimedia database. Every image is consisting of particular feature like color and shape. In database system, at back end there exits images dataset like medical imaging dataset. Here is need to define definition of multimedia, it is storage of datasets like medical images or other kind of images data set. Image database is not even browsing image but also to store image in database, retrieving digital images from the database after processing of particular features. Mainly the theme of this work is based on detection of image so content base image retrieval(CBIR) is also important to explore.

Storage of data is very important it is stored in different tables and their features are stored with some other tables so data is stored in two tables, one for images and one of features. Devised intelligent semantic has been used technique which has been discussed in coming section.

Different questions are raising regarding image retrieval are important. One very important question: how image will be retrieved efficiently. User find image from system through query in browser using features. Focus over functionality of query processing on back side but feature detection is important.

Work is under umbrella of semantics which find out relates different features. Semantics is defined on base of meta data. Technically, metadata data is defined at back end and query also executes at back end. Results of retrieval of query will be different tables of features. For example, if query: some steps have been called and features like shape, color and text involves inside image.

By implementing multimedia database, functionality is describing in steps, step one is to find out the query from user in cooperative environment then in second step name of image has been checked across image id during. In third step 'names of image' along image id is forwarded towards feature table, in this table every feature has a particular identification and check features table using image identification in every row of feature table. Feature table is consisting of group of features like across images name and image id. Therefore, process the information in comparative with help of feature table, related images are retrieved and this step is called query by feature and all relevant images are displayed. In step number four, every query calls particular image but every image has associated features so further related features are called. It means query by example has been called. Across every image, image feature is called there is exist a third table which bridge image table with feature table. For processing point of view need of study is to check content-based image retrieval because accuracy-based retrieval is required. From CBIR, result is feature table which shows feature with number of time occurred so find total occurrence of features in the images dataset and output is a table of extracted 
features. So such image retrieval from database is more accurate but from above text, it has been shown, feature detection is important for image retrieval.

This paper use study of different techniques of image processing like Harris corner detector, Lucas tracker, content-based image retrieval, first two techniques are good for feature detection and also more focus over scenario-based ontology. The main aim is feature detection in an image. Harris work on the basis of finding a point of interest whereas Kennard Lucas tracker take the difference between different image. These both two techniques are old technique but working for region of interest whereas content base image retrieval is based on knowledge representation which is domain of object detection. This technique finds the required image on the base of their features and content-based Image search system is essential for retrieving the accurate and relevant result of the image. Content based/feature-based image search based on the principles of semantics. Immediately question raises about semantics role here. The answer is a concept which is using for related image retrieval. Formally semantic retrieval is such search which is with reference to the context-based image search.

For understanding point of view, example scenario for feature detection like a cricket stadium/ brain tumor data set and when check semantic so features are called within image, when search this feature-based search so it's a rough search. But when user in interactive environment want to perform search over a particular feature of image, this type of search called a fine search. Multimedia database is such place from where data is extracting by using the Query be example, and also by query by feature. The space of this multimedia databases become increase as no. of features become increase in feature bases image search system. For optimization point of view, feature dimension must be reduced. In section number II description of literature review is present, in section III elaboration of proposed technique, section IV is based over issue and problem(limitation) as have seen and consider them as my future work.

\section{Literature Review}

A. Existing retrieval and detection of images. Harris corner detector detects the points of interest in an image [2], [4], such a detector identifies the invariant like rotation, scale, image noise. Detector is basically based on the auto correlation function of the signal. This function measures the local change in the whole image. So, it directs that algorithm find out the value of the interest on the basis of the interest point variants. For such identification ellipse shape, the elongation and size of the ellipse is given by the eigen values. The feature identification like edges, corner, and flat is made on the basis of the eigen values but this detection is for single feature, this identification is only based on corner or edge and only four points check intensity of image if features like in fifth side then how detection work.

Kennard Lucas tracker also works on image detection on the image registration [3]. Before this method number of technique are used, but they are fail in rotation of image and also other technique are much expensive as compare to KLT. KLT basically solved the image registration problem by having the function $\mathrm{f}(\mathrm{x})$ and $\mathrm{g}(\mathrm{x})$ and then these two functions give the respective pixel values at each location of $\mathrm{x}$ in each image. $\mathrm{X}$ is the vector. $\mathrm{H}$ is disparity vector. Which minimize the distance between $f(x+h)$ and $g(x)$, for some region of interest $R$. Registration algorithm is the way of problem solution? Because it gives the general way for the solution of the problem. Extended KLT have also been proposed [25] and when first frame get some drift then extended KLT retains position of templated in first template frame bur still problem persists if multiple features have been changed within frame.

For efficient feature extraction, there is a very important novel work has been performed [11-14]. This kind of extraction is automatic feature and, in such feature, are found after performing classification in image and this kind of classification is achieved from automatic segmentation. So, this paragraph has discussed about automatic features.

TABLE I. FEATURE EXTRACTION ACCURACY TABLE FROM LITERATURE

\begin{tabular}{|l|l|l|}
\hline Title & (Feature), (Method) & $\begin{array}{l}\text { Accuracy of Feature } \\
\text { Extraction }\end{array}$ \\
\hline Wang, N., Gao, X., Tao, D., and Li, X. (2014) & $\begin{array}{l}\text { (Color, edge), (Parametric shape } \\
\text { model/none parametric model) }\end{array}$ & Missing \\
\hline Piras, L., and Giacinto, G. (2017) & (Color), (Textual) & $88 \%$ \\
\hline $\begin{array}{l}\text { Rahim, M. S. M., Norouzi, A., Rehman, A., } \\
\text { and Saba, T. (2017) }\end{array}$ & (Shape, color), (Histogram) & $80 \%$ \\
\hline Kavitha, K., and Sudhamani, M. (2014) & (Color), (Harris detector) & $82.23 \%$ \\
\hline Ram, P., and Padmavathi, S. (2016) & (Color), (Harris detector) & $80 \%$ \\
\hline
\end{tabular}




\begin{tabular}{|l|l|l|}
\hline $\begin{array}{l}\text { Fernando, W., Udawatta, L., and Pathirana, P. } \\
(2007) .\end{array}$ & (Shape), (Lucas tracker) & Missing \\
\hline Mistry, S., and Patel, A. (2016) & (Shape), (Harris detector) & Missing \\
\hline $\begin{array}{l}\text { Fern, B. M., Rahim, M. S. M., Saba, T., } \\
\text { Almazyad, A. S., and Rehman, A. (2017) }\end{array}$ & (Shape), (EOH) & $96.02 \%$ \\
\hline $\begin{array}{l}\text { Despotović, I., Goossens, B., and Philips, W. W. } \\
\text { (2015) }\end{array}$ & (Shape, Color)(Histogram) & Missing \\
\hline
\end{tabular}

For feature retrieval two methods exists, parameter shaped based and none parameter shape-based method. Visual information retrieval has been improved with textual information in form of labels [7]. 3D bioinformation visualization treat disease as feature in form of shape and color [12]. features are categorized in form of local as well as global features and local features are retrieval has been achieved using Harris detector whereas global are extracts from color space so CBIR has improved [8]. features have been detected using edges in color space [4]. Lucas tracker algorithm is suing Taylor series which is iterative method for detection of feature [3]. Image acquisition and registration has performed; image registration is archived through Harris detector [2]. Classification also improve feature detection efficiently [8]. Hence accuracy compromise during retrieval of feature within CBIR system.

B. Other methods for feature detection/retrieval. Ontology is main core concept which provides the semantics for object description [1], [5], [8], [9], [16-21] With the concept of the semantics it is possible to find out the information with respect to the context. In simple words ontology provides the semantics. Ontology builds on the concepts of related attributes which belong to particular class. Like to build the application scenario of cricket if we want to develop the ontology of cricket so our ontology is for cricket so need to classify objects related cricket. Each class has particular attribute and also sub attributes. Each sub attribute is inheriting from particular super class attribute. Following defined ontology [1] gives the automatic ontology, can also be seen. It has been clear cut seen that CBIR detect feature by using color and shape histogram [25] but they are good for uniform feature and what should be mechanism for dynamic features.

So the problem formulate as followed: From well-known contribution it have been found that for $\mathrm{n}$ kind of images, $\mathrm{n}$ categories from those images in dataset compromise retrieval because features labeling(classification) is challenging even in one dataset. From reputed contribution it has been found that local features retrieval (harris corner detection, Bimodal Empirical mode decomposition) fill gap with automatic retrieval whereas global features retrieval(HSV) pertain manual retrieval and also comparison parameter like recall, precision also have degradation of features retrieval.This section motivate with different questions within system to detect features from different dataset of images and also within databased.

3. Methodology. Methodology is consisting of phase number one which supports CBIR but feature detection lacked and phase no. 2 is giving solution for multiple feature in phase 2 .

A. CBIR: Query based System Architecture (Phase no. 1). Architecture consists of four parts of the system, one is the user who do request of the related feature, second one is the query which will be process after the request of the user inside the system, third is the databases, and fourth one is the Browser where user request processing and can see the output. To explain below model Figure II.1 so an interface provided to the user from where user gives particular feature from user interface, with the selection of particular feature a query performs. The query had been forwarded towards database (DB) and results have been finding and terminology to be known as is known as query by feature the first query process and then call query by example. So, at last we have accessed DB, each feature regarding to image stored in the databases. From outside user query image which are based on particular features and those features based are displayed on the screen or the browser therefore process of feature extraction has been performed. 


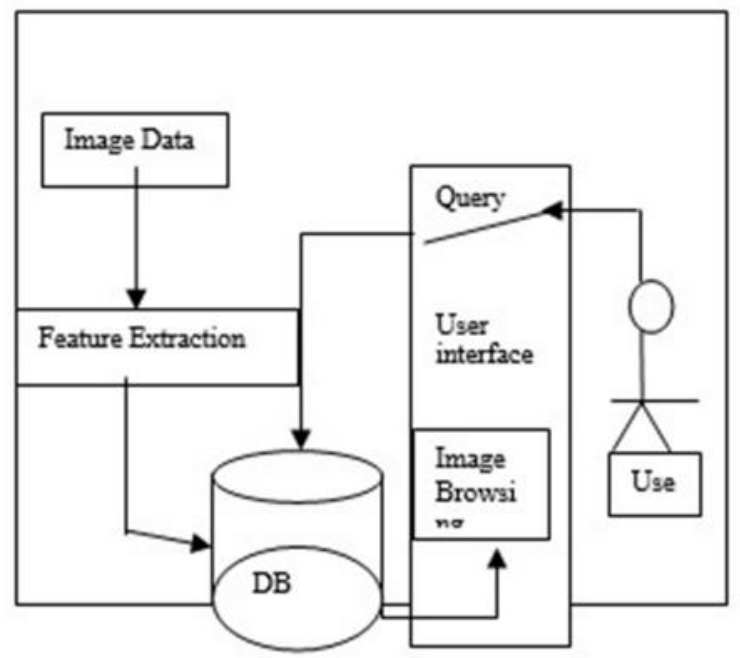

Fig. 1. : - Query based Feature work

The way in which I have described image based database, I can say that database is a Multimedia database. As number of images will stored inside images so length of multimedia databases also increases. It means to say that dimension of data is much high so flexibility exists to increase the length of images. The architecture of the Content based image search system is very much simple and easy to understand from user request up to extraction of image using the Multimedia databases. That is looking to be a simple database but in fact it is the multimedia databases as discussed. One more term which can be used here that is also called spatial media databases because images type data is spatial media data

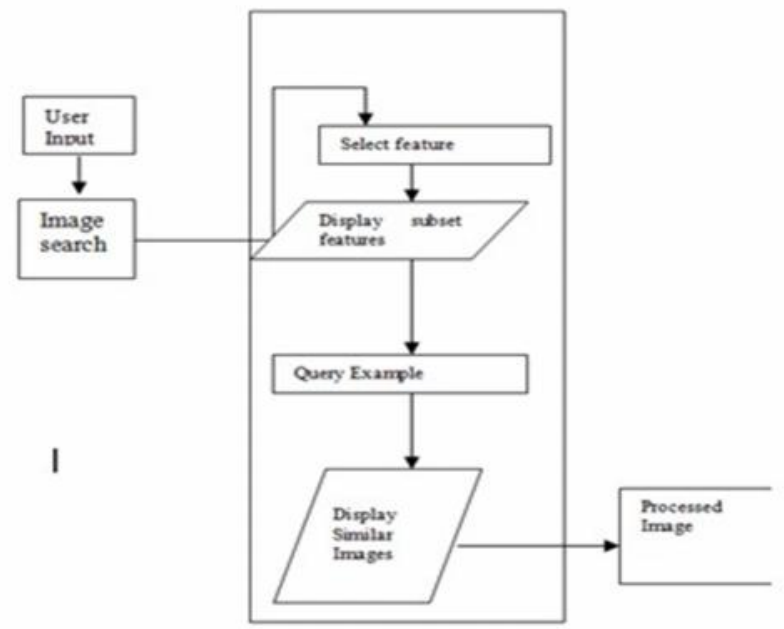

Fig. 2. : - Feature based query model

B. Features utilization of Query model. After getting motivation through the model, there is need to work some of model of feature based model. Which would be a dedicated algorithm.

Featured based Image ()

Step-1[Query is performed by user]

If Query $==1$

Go to step number 2

Else go to step number 5

Step-2[Selection of Feature]

IF (Feature match from image database)

Selection of feature is performed and go to step number 3

Else go to number 5 
Step-3 [Execution of Query model]

Step-4[Selection of subset features]

IF attached feature $==$ sub feature

Then display

Step number 5

Exit

These step of constructions gives the direction toward the implementation. This feature-based model Figure III.2 work is processed in five in steps, first step is selection of features initially, and after the selection of feature the query model would be executed. then the execution of query, you get subset of features. This subset feature based search is the rough search, and if you want to proceed the query model then select a particular image and perform a further search, this search is similarity-based search, it doesn't give the subset feature, it will give all superset features. That why it is called fine search. So, our end result is the processed search. Component of our system except the iterative refinement process. Steps of section 4 is main part of this paper. This is our main technique and proposed technique.

C. Query Based Image Retrieval. Here uses a new system technique component that is based on query. So new thing is the retrieval of image semantically and sequentially. The semantic retrieval of the image would be performed in two phases. First phase is query by feature and second phase is query by example. In above different section. These terms like Query by feature, query by example have been used before but cannot describe them in detail. So, let see them in following section.

D. Query by Feature. Query by feature is such search based on particular subset features and defending our above algorithm step number four, so all subset related images would be display by using this type of search? If searching query by feature so result is number of semantically found images: images with its corresponding degree of match $>0$, Ranked by degree of match. This ranked sequence is called "Feature sequence."

Multiple features query is also part of Query by feature: It Merges the corresponding feature sequence. This is Property of QBF known to be rough search.

E. Feature Description for an Image. Like metadata which figure III.3 describe the appearance of image. This makes QBF queries possible. Feature Description consists of some feature descriptors. Feature based description can be seen as follows: -

A ("feature value", "match level") pair

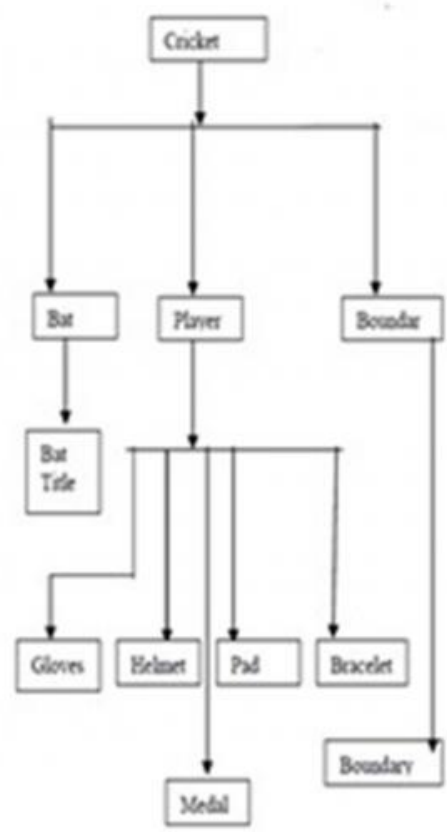

Fig. 3. - Ontology based Classification

F. Feature descriptor of similarity image base system. Match level is matching of feature and this question is important that how many features have been retrieved because this is more relative to accurate search. If the system is working with $\mathrm{N}$ feature images so methodology checks even single feature and also check that how much match 
level achieve. Match level is how many similar images for a particular feature. The whole work is performing on the basis of the feature description. So below table III.1 is showing the result of feature accurate extracted after processing of above algorithm.

TABLE II. ACCURATE SIMILARITY BASED IMAGE SEARCH

\begin{tabular}{|l|l|}
\hline Feature name & Match Level \\
\hline Bat & $30 / 40$ \\
\hline Bat Title & $16 / 40$ \\
\hline Bottle & $1 / 40$ \\
\hline Flag & $2 / 40$ \\
\hline Trophy & $1 / 40$ \\
\hline Wicket celebration & $18 / 40$ \\
\hline Umpire & $1 / 40$ \\
\hline Player Pad & $30 / 40$ \\
\hline Towel & $1 / 40$ \\
\hline Bowl & $12 / 40$ \\
\hline Camera Man & $1 / 40$ \\
\hline Collisions & $2 / 40$ \\
\hline Medal & $1 / 40$ \\
\hline Paper Cutting & $2 / 40$ \\
\hline Bracelet & $1 / 40$ \\
\hline
\end{tabular}

4. For Efficient Feature Detection Though Segmentation Classification. [27] In phase number 2, combination of DWT+PCA+KSVM with flavors (RBF, linear, Polygon kernels) has detected features within image 100 percent by

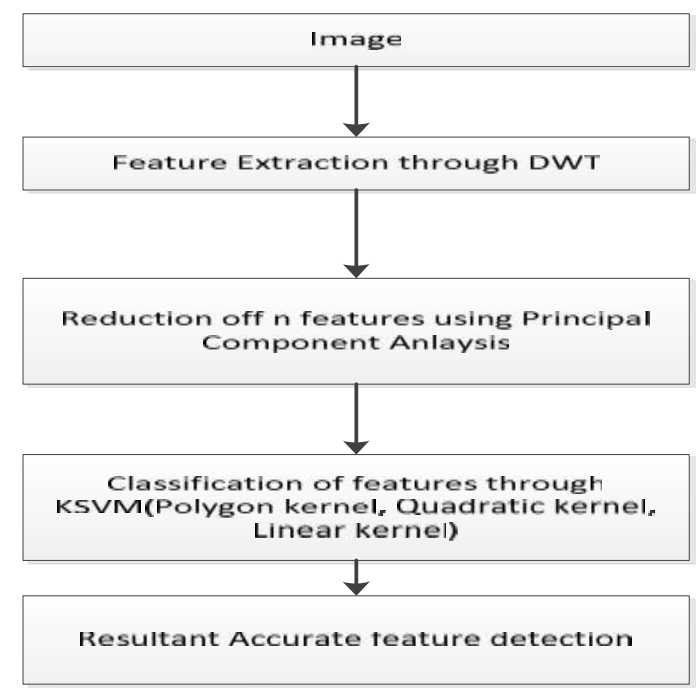

Fig. 4. Accurate Feature detection of CBIR

The image from dataset has been input to CBIR interactive system and CBIR system process dataset image, these data set images are further sending for accurate feature detection [27]. In step-1, [24][27] DWT (Discrete Wavelet transformation) is applying. Image is considering as a signal and presents in time domain. [22] In time domain image has been divided locally into bands so energy level of image features has been highlighted. 


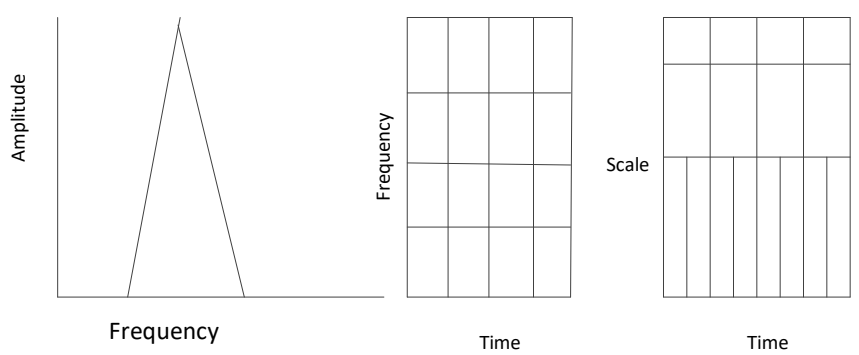

Fig.4. : - DWT signal representation

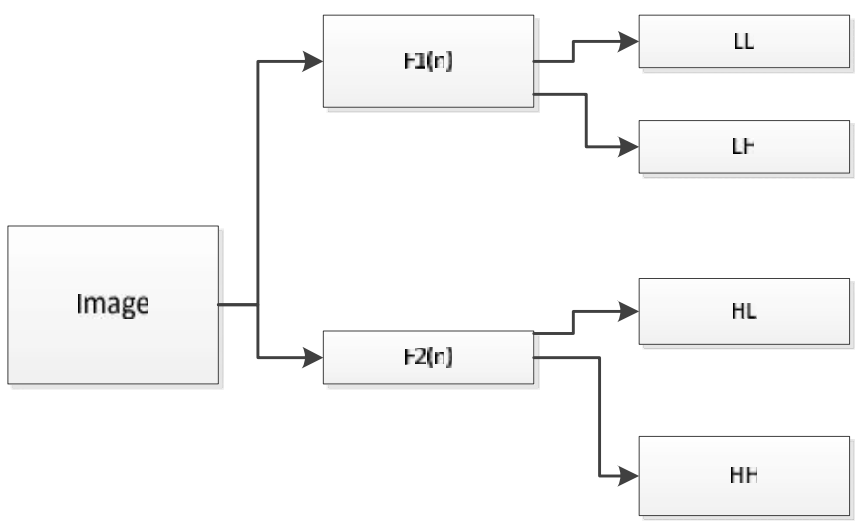

Fig. 5. DWT signal representation with high pass and low pass variations

In step number 2, PCA (Principal Component Analysis) performs for dimension reduction of image features. Firstly, identifies un correlated elements from image then least un correlated element remove from data set images so the group of most corelated elements have been obtained.

Step number three is accurate feature classification, obtained elements are classified through Support Vector Machine(SVM) like two classes are [-1,1]. SVM gives hyper plane and in such plan classified output(relevant) classes of images in query [23][].

$\mathrm{F}(\mathrm{x}, \mathrm{y})=\left\{\left(\mathrm{x}_{\left.\mathrm{n}, \mathrm{y}_{\mathrm{n}}\right)} / \mathrm{x}_{\mathrm{n}} \varepsilon \mathrm{R}^{\mathrm{p}}, \mathrm{y}_{\mathrm{n}} \varepsilon(1,-1)\right\}\right.$

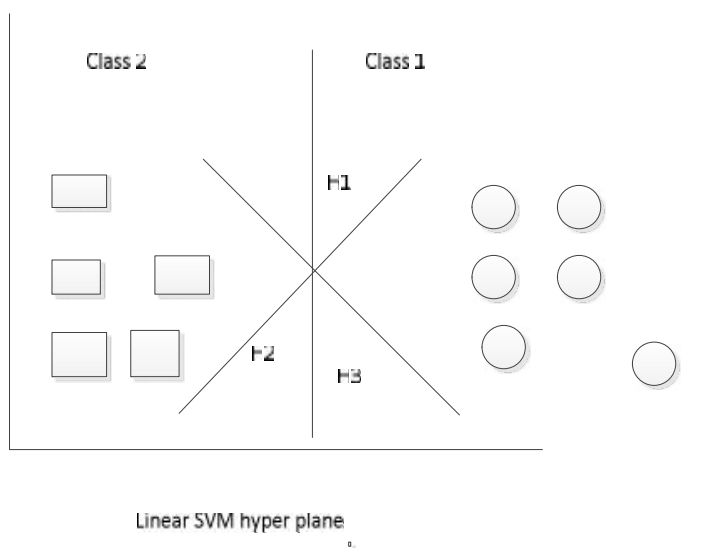

Fig. 6. SVM classification

KSVM has variation of SVM like RBF kernel, quadratic kernel and linear kernel for accurate detection of features in queried image. 


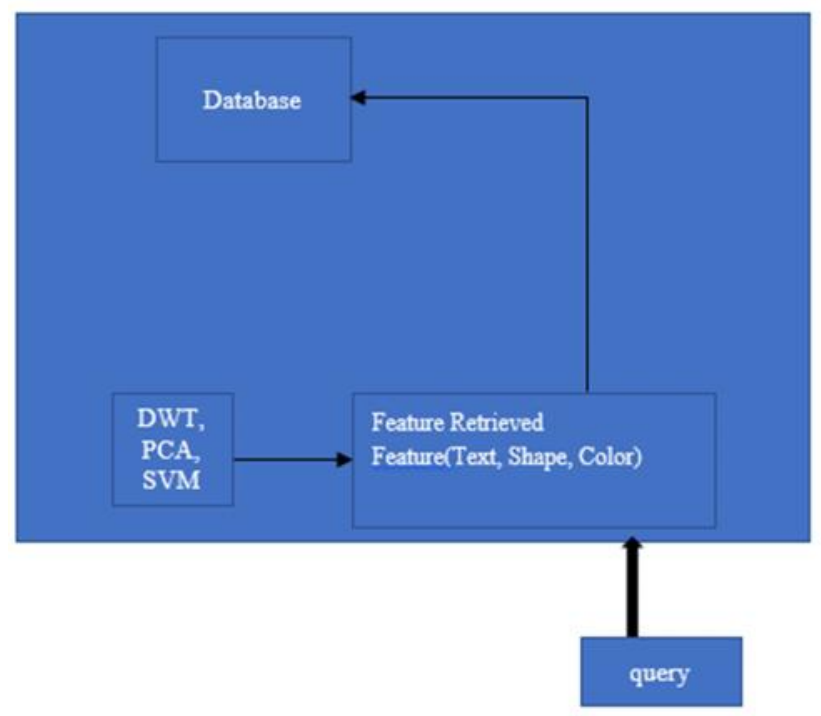

Fig. 7 Methodology diagram

Query has been performed from outside for efficient retrieval, from image their features like text, shape and color have been extracted with DWT, reduced with PCA because we need accuracy and reduced features are checked with SVM for further accurate decomposed features and they are matched with databased so retrieval of image from database have been efficiently improved.

5. Comparison With Other Image Based Search Systems And Limitation. Different image-based search systems have been explored and then some parameter has been defined and on the basis of those parameter the performance of this propose feature-based image retrieval can been compared. The parameters are Run time image databases update, Feature extraction, databases level, Query and also run time feature addition, each parameter will be checked against each and every system. So plan has compared every system one by one in form of numerical value as mentioned in given table for proposed system.

Conclusion. In this paper feature-based image retrieval become efficient with feature detection, the system worked on the basis of query system, and the query has been named as the query by feature and query by example but detection was lacked. Efficient retrieval has been possible with local and global reduced features (color and shape) with DWT, PCA and SVM like with accuracy of features. Future work should be on following aspects like improved CBIR will be designed in detail using segmentation and detection of image features will implement from different published repository like UCI, Harvard and segmentation values using combination of DWT+PCA+KSVM (kernel flavor) compared with other system on basis of segmentation methods.

\section{REFERENCES}

[1] Chmielewski, M., \& Stąpor, P. (2016). Medical data unification using ontology-based semantic model structural analysis. In Information Systems Architecture and Technology: Proceedings of 36th International Conference on Information Systems Architecture and Technology-ISAT 2015-Part III (pp. 139-151). Springer, Cham.

[2] Mistry, S., \& Patel, A. (2016). Image Stitching using Harris Feature Detection. International Research Journal of Engineering and Technology (IRJET), 3(04), 2220-6.

[3] Johansson, B. (2007). Derivation of Lucas-Kanade Tracker. In other words, 500, 7.

[4] Ram, P., \& Padmavathi, S. (2016, October). Analysis of Harris corner detection for color images. In Signal Processing, Communication, Power and Embedded System (SCOPES), 2016 International Conference on (pp. 405-410). IEEE.

[5] Mohanan, A., \& Raju, S. (2017). A Survey on Different Relevance Feedback Techniques in Content Based Image Retrieval. Int. Res. J. Eng. Technol, 4(2), 582-585. 
[6] Wang, N., Gao, X., Tao, D., Yang, H., \& Li, X. (2018). Facial feature point detection: A comprehensive survey. Neurocomputing, 275, 50-65.

[7] Iqbal, M., Naqvi, S. S., Browne, W. N., Hollitt, C., \& Zhang, M. (2016). Learning feature fusion strategies for various image types to detect salient objects. Pattern Recognition, 60, 106-120.

[8] Nakagawa, A., Kutics, A., Tanaka, K., \& Nakajima, M. (2003, September). Combining words and object-based visual features in image retrieval. In null (p. 354). IEEE.

[9] Kavitha, K., \& Sudhamani, M. V. (2014, January). Object based image retrieval from database using combined features. In Signal and Image Processing (ICSIP), 2014 Fifth International Conference on (pp. 161-165). IEEE.

[10] Piras, L., \& Giacinto, G. (2017). Information fusion in content based image retrieval: A comprehensive overview. Information Fusion, 37, 50-60.

[11] Piras, L., \& Giacinto, G. (2017). Information fusion in content based image retrieval: A comprehensive overview. Information Fusion, 37, 50-60.

[12] Rahim, M. S. M., Norouzi, A., Rehman, A., \& Saba, T. (2017). 3D bones segmentation based on CT images visualization.

[13] Iftikhar, S., Fatima, K., Rehman, A., Almazyad, A. S., \& Saba, T. (2017). An evolution based hybrid approach for heart diseases classification and associated risk factors identification. Biomedical Research, 28(8), 3451-3455.

[14] Rahim, M. S. M., Rehman, A., Kurniawan, F., \& Saba, T. (2017). Ear biometrics for human classification based on region features mining. Biomedical Research, 28(10).

[15] Mughal, B., Muhammad, N., Sharif, M., Saba, T., \& Rehman, A. (2017). Extraction of breast border and removal of pectoral muscle in wavelet domain. Biomedical Research, 28(11).

[16] Fern, B. M., Rahim, M. S. M., Saba, T., Almazyad, A. S., \& Rehman, A. (2017). Stratified classification of plant species based on venation state. Biomedical Research, 28(13), 5660-5663.

[17] Ejaz, K., Mateen, A., \& Ehsan, I. Vector Shape Classification and Z indexing.

[18] Lung, J. W. J., Salam, M. S. H., Rehman, A., Rahim, M. S. M., \& Saba, T. (2014). Fuzzy phoneme classification using multi-speaker vocal tract length normalization. IETE Technical Review, 31(2), 128-136.

[19] [18] Sharifara, A., Rahim, M. S. M., \& Bashardoost, M. (2013, September). A novel approach to enhance robustness in digital image watermarking using multiple bit-planes of intermediate significant bits. In Informatics and Creative Multimedia (ICICM), 2013 International Conference on (pp. 22-27). IEEE.

[20] Jabal, M. F. A., Rahim, M. S. M., Othman, N. Z. S., \& Jupri, Z. (2009, April). A comparative study on extraction and recognition method of cad data from cad drawings. In Information Management and Engineering, 2009. ICIME'09. International Conference on (pp. 709-713). IEEE.

[21] Harouni, M., Rahim, M. S. M., Al-Rodhaan, M., Saba, T., Rehman, A., \& Al-Dhelaan, A. (2014). Online Persian/Arabic script classification without contextual information. The Imaging Science Journal, 62(8), 437-448.

[22] Ejaz, K., \& Amjad, A. (2018). Model and Technique over Software Requirement Prioritization. Pakistan Journal of Engineering, Technology \& Science, 6(2).

[23] Z Zhang, Y. D., Chen, S., Wang, S. H., Yang, J. F., \& Phillips, P. (2015). Magnetic resonance brain image classification based on weighted-type fractional Fourier transform and nonparallel support vector machine. International Journal of Imaging Systems and Technology, 25(4), 317-327.

[24] Y Yang, G., Zhang, Y., Yang, J., Ji, G., Dong, Z., Wang, S., ... \& Wang, Q. (2016). Automated classification of brain images using wavelet-energy and biogeography-based optimization. Multimedia Tools and Applications, 75(23), 15601-15617.

[25] Zhang, Y., \& Wu, L. (2012). An MR brain images classifier via principal component analysis and kernel support vector machine. Progress In Electromagnetics Research, 130, 369-388.

[26] Oron, S., Bar-Hille, A., \& Avidan, S. (2014, September). Extended lucas-kanade tracking. In European Conference on Computer Vision (pp. 142-156). Springer, Cham.

[27] Liu, G. H., \& Yang, J. Y. (2013). Content-based image retrieval using color difference histogram. Pattern recognition, 46(1), 188-198. 
[28] Despotović, I., Goossens, B., \& Philips, W. (2015). MRI segmentation of the human brain: challenges, methods, and applications. Computational and mathematical methods in medicine, 2015. 